\title{
Capacitación: Evaluación áulica y desarrollo de habilidades docentes
}

\section{Training: Classroom assessment and teaching skills}

SÁNCHEZ-RIVERA, Lilia†** ESPERICUETA-MEDINA, Marta Nieves, MUÑOZ-LÓPEZ, Temístocles y DELGADO-ESTRADA, Sandra

Universidad Autónoma de Coahuila-Facultad de Ciencia, Educación y Humanidades

ID $1^{\text {er }}$ Autor: Lilia, Sánchez Rivera / ORC ID: 0000-0001-9468-2599, Researcher ID Tomson: T-1404-2018, CVU CONACYT ID: 613195

ID $1^{\text {er }}$ Coautor: Marta Nieves, Espericueta-Medina / ORC ID: 0000-0002-4924-4332, Researcher ID Tomson: T-15002018, CVU CONACYT ID: 372705

ID $2^{\text {do }}$ Coautor: Temístocles, Muñoz-Lopez / ORC ID: 0000-0003-4940-5730, Researcher ID Tomson: T-7834-2018, CVU CONACYT ID: 202437

ID $3^{\text {er }}$ Coautor: Sandra, Delgado Estrada / ORC ID: 0000-0002-7056-4843

DOI: $10.35429 /$ JET.2020.11.4.1.6

Recibido: 03 de Marzo, 2020; Aceptado 27 de Junio, 2020

Resumen

El objetivo de este articulo fue mostrar las necesidades de capacitación en evaluación áulica que inciden en las habilidades docentes de educación básica. La metodología que se utilizó fue cuantitativa y cualitativa, observacional, transversal y descriptiva y correlacional. La recopilación de la información fue a través de un cuestionario con reactivos en relación a necesidades de capacitación en evaluación áulica y las habilidades docentes. La muestra se conformó por 435 docentes de Educación Básica que laboran en 31 municipios del Estado de Coahuila de Zaragoza, el método de muestro fue no probabilístico por conveniencia, de acuerdo al diagnóstico de necesidades de capacitación de los docentes, realizado por la Secretaría de Educación del Estado de Coahuila. Las técnicas utilizadas fueron la estadística descriptiva, la multivariada y el análisis de datos textuales. De acuerdo a la evidencia empírica estadística se afirma que si los cursos de capacitación dotan a los docentes de estrategias que desarrollen la habilidad de un pensamiento creativo; por medio de elementos prácticos como lo son las técnicas de observación y de análisis de desempeño de los estudiantes impactará en su praxis de evaluación áulica.

Capacitación, Evaluación áulica, Habilidades docentes

\begin{abstract}
The objective of this article was to show the training needs in classroom evaluation that affect teaching skills. The methodology used was quantitative and qualitative, observational, cross-cutting and descriptive and correlation. The collection of information was through a questionnaire with reagents in relation to training needs in classroom evaluation and teaching skills. The sample consisted of 435 Basic Education teachers working in 31 municipalities of the State of Coahuila de Zaragoza, the sample method was non-probabilistic for convenience, according to the diagnosis of the teacher training necessities, performed by the Secretary of Education of the State of Coahuila. The techniques used were descriptive statistics, multivariate and analysis of textual data. According to statistical empirical evidence, it is stated that if training courses provide teachers with strategies that develop the skill of creative thinking; through practical elements such as observation techniques and student performance analysis will impact their classroom evaluation.
\end{abstract}

Training, Classroom assessment, Teaching skills

Citación: SÁNCHEZ-RIVERA, Lilia, ESPERICUETA-MEDINA, Marta Nieves, MUÑOZ-LÓPEZ, Temístocles y DELGADO-ESTRADA, Sandra. Capacitación: Evaluación áulica y desarrollo de habilidades docentes. Revista Teoría Educativa. 2020. 4-11: 1-6

\footnotetext{
* Correspondencia del Autor (Correo electrónico: 1sr14712@uadec.edu.mx)

$\uparrow$ Investigador contribuyendo como primer autor.
} 


\section{Introducción}

Actualmente, el rol del docente ha tenido que incorporar diversas estrategias de evaluación áulica, acciones que han insidido en sus habilidades en la praxis educativa; en este sentido resulta importante que se les otorgue capacitación para que desarrolle con más eficiencia la recogida de evidencia del aprendizaje en el contexto que se ejerce. El presente estudio busca mostrar las necesidades de capacitación en evaluación áulica que inciden en las habilidades docentes. Con base a lo anterior proponer acciones que ayuden al ejercicio de la acción docente y ser un actor educativo que impacte en el estudiante de "aprender cómo aprender".

Si se entiende que "enseñar es ayudar a aprender", es necesario reflexionar sobre la forma en que se aprende (el cómo), para poder actuar, así, en consecuencia (Román, 1995). Desde el punto de vista pedagógico, se trata de tener en cuenta factores que tienen un papel importante en el proceso de aprendizaje del alumno y que dan origen a diferencias individuales importantes, que los docentes deben considerar a la hora de ajustar su enseñanza (Castellá, 2007; Martínez, 2007). Por ello, el personal docente, para responder a las actuales transformaciones educativas, debe actualizarse, formarse y capacitarse tanto en aspectos disciplinares como pedagógicos, ya que los cambios permanentes, a su vez, originan el ejercicio de roles diferentes a los desempeñados en las décadas anteriores. Asimismo, las formas de la organización y estructura de los currículos se han flexibilizado de tal manera que habrá que adaptarse ellas, con elementos acordes para ofrecer mejores niveles de calidad en la formación educativa. Esto nos lleva a reflexionar sobre las necesidades de capacitación o superación que tienen los grupos docentes como uno de los elementos clave para responder a los desafíos y demandas actuales de la educación. (Vélez Chablé, 2006 citado por Zuñiga, 2020, p.8).

Chehaybar (2007) citado por Guambo (2020) señala que “...la formación docente es un proceso permanente, dinámico, integrado, multidimensional; entonces la disciplina y sus aspectos teóricos, metodológicos, epistemológicos, didácticos, psicológicos, y sociales, son elementos que convergen para lograr la profesionalización de la docencia". ( $p$ 408).
En relación con la capacitación Chiavenato (2009) señala que, "La capacitación es el proceso educativo de corto plazo, aplicado de manera sistemática y organizada por medio del cual las personas adquieren conocimientos desarrollan habilidades y competencias en función de objetivos específicos". (p. 312).

Adicionalmente, (Lavigne, 2013, citada por Guambo, 2020, p 409) expresa: los docentes deben tener la preparación para atender las necesidades de los estudiantes de medios desfavorecidos, incluidos los que asisten a escuelas que se encuentran en zonas remotas o tienen recursos escasos. Los docentes necesitan capacitación en el uso de instrumentos de evaluación que les permitan detectar y abordar las dificultades de aprendizaje en una etapa temprana. (p. 5).

Ahora bien, de acuerdo al ministerio de educación pública citado por Zuñiga (2020) menciona que:

La evaluación permite ... documentar y valorar las características y avance del estudiantado en el desarrollo de habilidades para una nueva ciudadanía. Cuando el estudiantado, socializa sus ideas previas, representa una evaluación diagnóstica sobre sus conocimientos y habilidades iniciales. Al continuar con la secuencia de situaciones de aprendizaje, se hace énfasis en el trabajo colaborativo, resolución de problemas presentes en la comunidad, se discuten y contrastan los hallazgos, y vuelven a revisar sus primeras ideas, lo que le permite conocer cómo ha evolucionado en su aprendizaje, considerando los aspectos atinentes a la evaluación formativa y sumativa. (p.10).

Las necesidades actuales conllevan al docente a realizar estrategias de evaluacion en linea a cordes al aprendizaje esperado; en este sentido González (2008) citado por Zeballos (2020) señala que, "en la formación online hay que tener presente que la evaluación debe estar correctamente combinada y compensada con otros aspectos fundamentales para asegurar el éxito de la acción formativa". (p.84).

Lo ideal, según el esquema de Hall y Hord (2006), sería crear condiciones que llevan los docentes a un nivel de uso de refinamiento como una norma y práctica profesional colectiva de mejoramiento continuo. 
Es necesario resaltar la importancia que tiene la capacitación en la formación docente, la cual les permita sopesar sobre su propia práctica para la mejora de sus habilidades docentes que impactan en sus procesos de enseñanzaaprendizaje.

De ahí que, el presente artículo da cuenta de la acciones de capacitación en la formación docente de educación básica del estado de Coahuila de Zaragoza, en relación al curso- taller que tuvo como objetivo que los docentes analizaran y aplicaran las bases y las herramientas de evaluación áulica y habilidades docentes; además de que identificaran y mostraran sus necesidades de capacitación. Asimismo, determinar el impacto que tuvo en los participantes el curso-taller y la calidad del proceso formativo desarrollado e implementado.

\section{Metodología a desarrollar}

Al término del curso-taller, se aplica una encuesta a 435 docentes en línea y en físico para los casos que no les fue posible contar con un área adecuada para dar respuesta en línea; consta de cinco apartados: el primero de ellos refiere a los datos generales, el segundo mide la experiencia del docente, la tercera mide al capacitador, la cuarta evalúa al curso-taller y el último apartado otorga datos textuales en relación a los temas abordados.

La base de datos se explora desde un análisis cuantitativo y cualitativo. Cuantitativo: análisis descriptivo desde frecuencias y porcentajes para caracterizar a la muestra y análisis multivariado desde el factorial exploratorio; cuyo objetivo es observar las variables que subyacen del fenómeno de estudio.

Finalmente, se lleva a cabo un análisis cualitativo de datos textuales para conformar la redes semánticas; las cuales son una opción metodológica para acercarse al significado de un concepto de un colectivo. Se solicita al colectivo mencionar 5 palabras que asociaría libremente con el estímulo de: necesidades de capacitación, evaluación áulica, conceptos más significativos para su aprendizaje para el desarrollo de habilidades y estrategias áulicas a implementar en su entorno laboral, una vez anotadas las palabras se les indicó asignar a cada palabra un número que va de menor a mayor, donde el 1 significa que es el más cercano al término presentado y 5 el menos cercano al término.
Tamaño de la red refiere a la cantidad total de palabras definidoras que se utilizaron para describir los conceptos cuestionados, en relación con el peso semántico se observa las palabras definidoras de los conceptos, de acuerdo al valor de cada definidora obtenido por la frecuencia y la ponderación dada por cada caso.

Para determinar el núcleo de la red se siguió el procedimiento propuesto por Reyes (1993) donde sugiere que su delimitación se sustenta en el punto de quiebre (scree-Test) propuesto por cattell (1952, citado por Reyes, 1993). Se grafican todos los pesos semánticos en forma descendiente y se realiza el corte cuando la pendiente de la curva adquirió un carácter asintótico en relación con el eje de la X“s.

De lo anterior se desarrolla la hipotesis alternativa de:

Hi. La capacitación en evaluación áulica inciden en las habilidades docentes de educación básica.

\section{Resultados}

\section{Estadística descriptiva}

1.1. Análisis de frecuencia y porcentaje

En este apartado se da a conocer el análisis descriptivo, a través de frecuencias y porcentajes con el propósito de caracterizar a la población encuestada en relación al curso-taller; siendo 435 docentes de Educación Básica que laboran en 31 municipios del Estado de Coahuila de Zaragoza; donde se destaca que el municipio de Torreón registra la mayor asistencia (n: 96, 23.4\%), le sigue Saltillo con (n: 59,14.4\%), continúan Piedras Negras y Frontera con (n: 28, $6.8 \%$ ), cabe mencionar que el resto de los 11 municipios reportan la participación más baja.

2. Análisis multivariado

2.1 Factorial exploratorio

La mayoría de los valores adjudicados a los atributos por los docentes asistentes al cursotaller están en rangos altos, por lo que se realiza un análisis más fino que muestre la dispersión real de las puntuaciones. 
El análisis factorial permite discriminar mejor los valores, se utiliza el criterio de corte que sugiere el programa (carga factorial $\geq 0.70$ ) que se traduce en la máxima variabilidad de la probabilidad $(\mathrm{p}=\mathrm{p} / \mathrm{q}=0.5 / 0.5)$ de $\mathrm{R}^{2}$. Con este criterio se extrajeron cinco factores, donde se denotan variables más relevantes son en los factores 1,3 y 4 y se anulan las variables que tuvieron valor $\leq 0.70$.

Los factores son denominados así de acuerdo a las variables con cargas factoriales significativas que los constituyen.

En el Factor 1 Capacitación. La capacitación del curso-taller se destaca la ejecución, el control y la organización de sus actividades; además que alcanzó el objetivo general por poseer contenidos acordes a la temática con un tiempo pertinente en cada bloque, cubrió las expectativas del participante por los temas abordados de evaluación y habilidades docentes, conjuntamente mostró las distintas posibilidades que posé el participante para llevar acabo los temas de acuerdo al Programa de Profesionalización Docente.

En el Factor 3 Habilidades docentes, En el curso-taller el docente adquirió nuevas habilidades prácticas que puede aplicarlas en su entorno, cubrió sus expectativas por sistematizar su pensamiento creativo. Al mismo tiempo que con los temas vistos se alcanzó el objetivo general.

En Factor 4 Evaluación áulica, se observa que el curso-taller apoyo a sistematizar el pensamiento creativo de los participantes a partir de que se modelaron habilidades de instrumentalización de la observación y la evaluación áulica con prácticas exposiciones, lenguaje apropiado y temas abordados adecuados que enriquecieron temáticas sobre la evaluación.

\section{Análisis complementarios}

3.1 Redes semánticas de las necesidades de capacitación

Para la observación de los datos el tamaño de la red refiere la cantidad de palabras que utilizaron los docentes para describir las necesidades de capacitación mismas que representan la riqueza de la red que en este caso fue de 320 palabras definidoras que se dieron al estímulo.
En relación con el peso semántico, las palabras definidoras de necesidades de capacitación siendo las de mayor peso semántico: planeación, diseño de estrategias, evaluación, matemática, inclusión, seguidas por la necesidad de conocer más sobre evaluación áulica, así como cuestiones relacionadas con el uso de material didáctico, adecuaciones curriculares, motivación, neuroeducación, lecto escritura, neurociencia y comunicación asertiva.

\subsection{Red semántica de evaluación áulica}

La red que en este caso fue de 294 palabras definidoras, resultando con mayor peso semántico del concepto de evaluación áulica: análisis, habilidades, reconocimiento, jerarquizar ideas, objetiva, observación, resúmenes y evaluación.

3.3 Red semántica de conceptos significativos durante el curso

Los docentes mencionaron en primer lugar la importancia de la observación y sus características seguida de herramientas para evaluar, asimismo, se encontró que de acuerdo a su peso semántico las palabras necesarias para definir el concepto son 14 de 270 del total de la red.

3.4 Red semántica de estrategias áulicas para implementar en su área laboral

Se encontró que las palabras necesarias para definir el concepto estrategias áulicas para implementar en su área laboral son 12 de 300 de acuerdo a su peso semántico. Los participantes mencionaron en primer lugar los instrumentos y su aplicación, enseguida las rubricas, registro anecdótico, tabla de actitudes, bitácoras, diario de clase, estudio de caso, ABP, y lista de cotejo.

\section{Conclusiones}

Con base en los resultados obtenidos de los analisis estadísticos presentados, se exponen las conclusiones que muestran la perspectiva de capacitación: evaluación áulica y desarrollo de habilidades docentes. Para dar respuesta al objetivo planteado y la hipotesis alternativa planteada se concluye lo siguiente: 
Con la capacitación otorgada a los docentes de educación básica se desarrollaron nuevas habilidades prácticas, se sistematizó su pensamiento creativo, en relación a la evaluación áulica adquirieron habilidades sobre la instrumentalización de la observación y la evaluación con ejercicios prácticos.

Las necesidades de capacitación que externaron los docentes principalmente son en planeación, diseño de estrategias, evaluación, matemática, inclusión, seguidas por la necesidad de conocer más sobre evaluación, así como cuestiones relacionadas con el uso de material didáctico, adecuaciones curriculares, motivación, neuroeducación, lectoescritura, neurociencia y comunicación asertiva.

La conceptualización que tienen los docentes en relación a la evaluación áulica es lo relacionado con el análisis, habilidades, reconocimiento, jerarquizar ideas, objetiva, observación, resúmenes y evaluación.

Por su parte las estrategias áulicas para implementar en su área laboral, los docentes mencionaron, rubricas, registro anecdótico, tabla de actitudes, bitácoras, diario de clase, estudio de caso, aprendizaje basado en problemas (ABP) y lista de cotejo.

Se debe de entender que la evaluación áulica es vista desde cualquier contexto de aprendizaje, es decir, lo antes dicho ha servido para llevar acabo la evaluación y el desarrollo de habilidades, sin embargo; es importante destacar que el entorno actual generado por pandemia del coronavirus 19, abre una brecha de investigación en el cual, el uso de las tecnologías juega un papel protagónico.

Ante esta contingencia sanitaria y con base a la nueva normalidad surge la necesidad de realizar e implementar proyectos innovadores que den solución a las necesidades académicas de todos los niveles.

Es así, que las nuevas necesidades de capacitación deben de estar encaminadas al uso de las Tecnologías de la Información y la Comunicación (TIC) para que, de manera conjunta con las habilidades ya desarrolladas impacten en los aprendizajes esperados.

\section{Referencias}

Avila Carvajal, A. A., \& Valarezo Castro, J. W. (2020). Perfiles de autorregulación en cursos online. Machala:Editorial UTMACH.

Castellá, J. M., Comelles, S., Cros, S. y Vilá, M. (2007). Entender(se) en clase: Las estrategias de los docentes bien valorados. Barcelona: GRAO.

Chehaybar, E. (2007). Reflexiones sobre el papel del docente en la calidad educativa. Reencuentro, (50), 100-106.

Chiavenato, I. (2009). Gestión del talento Humano. México, D.F.: McGraw Hill.

Guambo, Q., \& del Rosario, D. (2020). Formación didáctica del docente para la atención a la diversidad en cuarto año de Educación General Básica (Master's thesis, Guayaquil: ULVR, 2020.).

Hall, Gene E. \& Hord, Shirley M. (2006). Implementing change: Patterns, principles, and pothole (Second Edition). Needham Heights, MA: Allyn \& Bacon. (Purchase at Bob Miller Book Room, 180 Bloor St. W. Lower Concourse)

Llivina Lavigne, M. J. y Urrutia Romaní, I. (2013). La formación de un docente de calidad para el desarrollo sostenible. Programa de Educación. Unesco. Obtenido de: http://www.unesco.org/new/fileadmin/MULTI MEDIA/FIELD/Havana/pdf/Formaciondocente s_Llivina.pdf

Martínez Geijo, P. (2007). Aprender y enseñar: Los estilos de aprendizaje y de enseñanza desde la práctica de aula. I.C.E.

Reyes Lagunes, I. (1993). Las redes semánticas naturales, su conceptualización y su utilización en la construcción de instrumentos. Revista de Psicología Social y Personalidad, 10(1), 81-97.

Román, J. M. (1995). Métodos de enseñanza. En J. Beltrán, y J. A. Bueno Álvarez (Ed.), Psicología de la educación (p. 479-527). 
Sarobe, M., Russo, C. C., Ahmad, T., La Riva, D. D., \& Traverso, P. (2020). Impacto del Plande Continuidad Académica en la situación de emergencia sanitaria COVID-19 de la UNNOBA. In XV Congreso Nacional de Tecnología en Educación y Educación en Tecnología (TE\&ET 2020)(Neuquén, 6 y 7 de julio de 2020).

Vélez Chablé, G. (2006). El docente ante los retos educativos del siglo XXI. Pampedia, 2, 5559. https:// www.academia.edu/20417364/6-Eldocente-ante-los-retos-educativos-del-siglo-

XXI.

Zeballos, M. (2020). La evaluación de los aprendizajes mediadas por las TAC. Revista Tecnológica-Educativa Docentes 2.0, 9(1), 8395.

Zúñiga-Meléndez, A., Durán-Apuy, A., Chavarría-Vásquez, J., Gamboa-Araya, R., CarballoArce, A. F., Vargas-González, X., \& Torres-Salas, I. (2020). Diagnóstico de las necesidades de capacitación de docentes de biología, química, física y matemática, en áreas disciplinares, pedagógicas, y uso de las tecnologías para la promoción de habilidades de pensamiento científico. Revista Electrónica Educare, 24(3), 1-32 